\title{
Nonlinear Singular BVP of Limit Circle Type and the Presence of Reverse-Ordered Upper and Lower Solutions
}

\author{
Amit K. Verma ${ }^{1}$ and Lajja Verma ${ }^{2}$ \\ ${ }^{1}$ Department of Mathematics, BITS Pilani, Pilani, Rajasthan 333031, India \\ ${ }^{2}$ Department of Mathematics and Astronomy, University of Lucknow, Lucknow 226007, India
}

Correspondence should be addressed to Amit K. Verma, amitkverma02@yahoo.co.in

Received 27 May 2011; Accepted 3 July 2011

Academic Editor: Mohamed A. El-Gebeily

Copyright (C) 2011 A. K. Verma and L. Verma. This is an open access article distributed under the Creative Commons Attribution License, which permits unrestricted use, distribution, and reproduction in any medium, provided the original work is properly cited.

We consider the following class of nonlinear singular differential equation $-\left(p(x) y^{\prime}(x)\right)^{\prime}+$ $q(x) f\left(x, y(x), p(x) y^{\prime}(x)\right)=0,0<x<1$ subject to the Neumann boundary condition $y^{\prime}(0)=$ $y^{\prime}(1)=0$. Conditions on $p(x)$ and $q(x)$ ensure that $x=0$ is a singular point of limit circle type. A simple approximation scheme which is iterative in nature is considered. The initial iterates are upper and lower solutions which can be ordered in one way $\left(v_{0} \leq u_{0}\right)$ or the other $\left(u_{0} \leq v_{0}\right)$.

\section{Introduction}

The upper and lower solution technique is the most promising technique as far as singular boundary value problems, are concerned [1]. Recently, lot of activities are there regarding upper and lower solutions technique (see $[2,3]$ and the references therein). To see the application of the similar kind of problems, one should see the references of [3]. In most of the results, upper and lower solutions are well ordered, that is, $u_{0} \geq v_{0}$. As far as reverse-ordered upper and lower solutions are considered, that is, $u_{0} \leq v_{0}$, the literature is not that rich. Though references are there for nonsingular boundary value problem, but singular boundary value problems require further exploration. The details of the work done for the nonsingular problem when upper and lower solutions are in reverse order can be seen in $[4,5]$. To fill this gap in the present paper, we consider the following singular BVP:

$$
\begin{gathered}
-\left(p(x) y^{\prime}(x)\right)^{\prime}+q(x) f\left(x, y(x), p(x) y^{\prime}(x)\right)=0, \quad 0<x<1, \\
y^{\prime}(0)=0, \quad y^{\prime}(1)=0 .
\end{gathered}
$$


(A1) Let $p(x)$ satisfy the following conditions.

(i) $p(0)=0$ and $p>0$ in $(0,1)$.

(ii) $p \in C[0,1] \cap C^{1}(0,1)$.

(A2) Let $q(x)$ satisfy the following conditions.

(i) $q(x)>0$ in $(0,1)$ and $q(x) \in C(0,1]$.

(ii) $\int_{0}^{1} q(x) d x<\infty$.

(iii) $\lim _{x \rightarrow 0}(q(x)) /\left(p^{\prime}(x)\right)=0$.

(iv) $\int_{0}^{1}(1 / p(x))\left(\int_{0}^{x} q(s) d s\right)^{1 / 2} d x<\infty$.

In this paper, we consider a computationally simple iterative scheme defined by

$$
\begin{gathered}
-\left(p y_{n}^{\prime}\right)^{\prime}+\lambda q y_{n}=-q f\left(x, y_{n-1}, p y_{n-1}^{\prime}\right)+\lambda q y_{n-1}, \quad 0<x<1, \\
y_{n}^{\prime}(0)=0, \quad y_{n}^{\prime}(1)=0 .
\end{gathered}
$$

Starting with upper and lower solutions, we generate monotone sequences. To generate these monotonic sequences, we need the existence of some differential inequalities. To prove these differential inequalities, we analyze the corresponding singular IVP and extract properties of the solutions and their derivative.

We have arranged the paper in four sections. In Section 2, we discuss some elementary results, for example, maximum principles and existence of two differential inequalities. Then using these elementary results, we establish existence results for well-ordered upper and lower solutions in Section 3 and for reverse-ordered upper and lower solutions in Section 4. In Section 5, we conclude this paper with some remarks.

\section{Preliminaries}

Let $h(x) \in C[0,1]$, and let $\lambda \in \mathbb{R}_{0}\left(\mathbb{R}_{0}=\mathbb{R} \backslash\{0\}\right)$, let $A \in \mathbb{R}$ and let $B \in \mathbb{R}$. Now, consider the following class of linear singular problems:

$$
\begin{gathered}
-\left(p(x) y^{\prime}(x)\right)^{\prime}+\lambda q(x) y(x)=q(x) h(x), \quad 0<x<1, \\
y^{\prime}(0)=A, \quad y^{\prime}(1)=B .
\end{gathered}
$$

The corresponding homogeneous system (eigenvalue problem) is given by

$$
\begin{gathered}
-\left(p(x) y^{\prime}(x)\right)^{\prime}+\lambda q(x) y(x)=0, \quad 0<x<1, \\
y^{\prime}(0)=0, \quad y^{\prime}(1)=0 .
\end{gathered}
$$


The solution of the nonhomogeneous problem (2.1)-(2.2) can be written as follows:

$$
w(x)=z_{1}(x)\left[\int_{0}^{x} \frac{q(t) h(t) z_{0}(t)}{W_{p}\left(z_{1}, z_{0}\right)} d t+\frac{A}{z_{1}^{\prime}(0)}\right]+z_{0}(x)\left[\int_{x}^{1} \frac{q(t) h(t) z_{1}(t)}{W_{p}\left(z_{1}, z_{0}\right)} d t+\frac{B}{z_{0}^{\prime}(1)}\right]
$$

where $z_{0}(x, \lambda)$ is the solution of

$$
-\left(p(x) z_{0}^{\prime}(x)\right)^{\prime}+\lambda q(x) z_{0}(x)=0, \quad 0<x<1, z_{0}(0)=1, z_{0}^{\prime}(0)=0,
$$

$z_{1}(x, \lambda)$ is the solution of

$$
-\left(p(x) z_{1}^{\prime}(x)\right)^{\prime}+\lambda q(x) z_{1}(x)=0, \quad 0<x<1, z_{1}(1)=1, z_{1}^{\prime}(1)=0
$$

and $W_{p}\left(z_{1}, z_{0}\right)=p(t)\left(z_{1} z_{0}^{\prime}-z_{1}^{\prime} z_{0}\right)$. By replacing $x$ with $1-x$ in (2.6), it is easy to verify that

$$
z_{1}(x)=z_{0}(1-x)
$$

for both positive and negative values of $\lambda$.

Remark 2.1. Existence of $z_{0}(x)$ and $z_{1}(x)$ satisfying the IVP (2.6) and IVP (2.7), respectively, is an immediate consequence of the result due to $\mathrm{O}^{\prime}$ Regan [6, Theorem 2.1, page 432].

Remark 2.2. Let $L_{q}^{2}(0,1)$ be a Hilbert space with inner product defined by

$$
\langle f, g\rangle=\int_{0}^{1} q(x) f(x) \overline{g(x)} d x
$$

From (A2) (iv), it can easily be verified that $x=0$ is a singular point of limit circle type (see [6, Remark (i) page 434]) in $L_{q}^{2}(0,1)$. Thus, we have pure point spectrum [7, page 125]. It is easy to show that the eigenvalues are real, simple, and negative.

Remark 2.3. Since $z_{0}$ and $z_{1}$ are two linearly independent solutions of (2.3), the eigenvalues of the eigenvalue problem (2.3)-(2.4) will be the zeros of $z_{0}^{\prime}(1, \lambda)$. Since $z_{0}^{\prime}(1, \lambda)$ is an analytic function of $\lambda$ so its zeros will be isolated and they all will be negative. Let them be $-\lambda_{0},-\lambda_{1},-\lambda_{2}, \ldots$, where $\lambda_{i}>0$ for $i=0,1,2, \ldots$. Now, we have $-\lambda_{0}$ as the first negative zero of $z_{0}^{\prime}(1, \lambda)$ or in other words first negative eigenvalue of (2.3)-(2.4).

Since $z_{0}(x, \lambda)$ does not change its sign for $-\lambda_{0}<\lambda<0$ and $z_{0}(0, \lambda)=1$; therefore, $z_{0}(x, \lambda)>0$ for all $x \in[0,1]$ and for all $-\lambda_{0}<\lambda<0$.

Remark 2.4. Using (2.6), $z_{1}(x)=z_{0}(1-x)$, it is easy to prove that if $\lambda>0$ then for all $x \in(0,1]$, $z_{0}(x)>1$, and $z_{0}^{\prime}(x)>0$ and for all $x \in[0,1)$, we have $z_{1}(x)>1$ and $z_{1}^{\prime}(x)<0$.

Remark 2.5. Using Remark 2.3, $z_{1}(x)=z_{0}(1-x)$, and the differential equation (2.6), it is easy to prove that if $-\lambda_{0}<\lambda<0$ for all $x \in[0,1), z_{0}(x)>0$ and $z_{1}^{\prime}(x)>0$ and for all $x \in(0,1]$, we have $z_{0}^{\prime}(x)<0$ and $z_{1}(x)>0$. 
Remark 2.6. Let $\lambda>0$ and let $h \in C[0,1]$. If $h \geq 0$ (or $h \leq 0$ ), then

$$
\int_{0}^{x} \frac{q(t) h(t) z_{0}(t)}{W_{p}\left(z_{1}, z_{0}\right)} d t, \quad \int_{x}^{1} \frac{q(t) h(t) z_{1}(t)}{W_{p}\left(z_{1}, z_{0}\right)} d t
$$

are nonnegative (or nonpositive).

Remark 2.7. Let $-\lambda_{0}<\lambda<0$ and let $h \in C[0,1]$. If $h \geq 0$ (or $h \leq 0$ ), then

$$
\int_{0}^{x} \frac{q(t) h(t) z_{0}(t)}{W_{p}\left(z_{1}, z_{0}\right)} d t, \quad \int_{x}^{1} \frac{q(t) h(t) z_{1}(t)}{W_{p}\left(z_{1}, z_{0}\right)} d t
$$

are nonpositive (or nonnegative).

Proposition 2.8 (Maximum Principle). Let $\lambda>0$. If $A \leq 0, B \geq 0$ (or $A \geq 0, B \leq 0$ ) and $h \in C[0,1]$ is such that $h \geq 0$ (or $h \leq 0)$, then $w(x) \geq 0($ or $w(x) \leq 0)$, where $w(x)$ is the solution of (2.1)-(2.2).

Proposition 2.9 (Antimaximum Principle). Let $-\lambda_{0}<\lambda<0$. If $A \leq 0, B \geq 0$ (or $\left.A \geq 0, B \leq 0\right)$ and $h \in C[0,1]$ is such that $h \geq 0$ (or $h \leq 0)$, then $w(x) \leq 0$ (or $w(x) \geq 0)$, where $w(x)$ is the solution of (2.1)-(2.2).

Now, we derive conditions on $\lambda$ which will help us to prove the monotonicity of the solutions generated by the iterative scheme (1.2).

Lemma 2.10. Let $M$ and $N \in \mathbb{R}^{+}$. If $\lambda>0$ is such that

$$
\lambda \geq M\left(1-N \int_{0}^{1} q(x) d x\right)^{-1}
$$

then for all $x \in[0,1]$,

$$
(M-\lambda) z_{0}(x)+N p(x) z_{0}^{\prime}(x) \leq 0 .
$$

Proof. Integrating (2.6) from 0 to $x$ and using the fact that $z_{0}^{\prime}(x)>0$ in $(0,1]$, we get

$$
p(x) z_{0}^{\prime}(x) \leq \lambda z_{0}(x) \int_{0}^{1} q(x) d x
$$

Therefore, we get $(M-\lambda) z_{0}(x)+N p(x) z_{0}^{\prime}(x) \leq(M-\lambda) z_{0}+N \lambda z_{0}(x) \int_{0}^{1} q(x) d x$. Hence, (2.13) will hold if $(M-\lambda)+N \lambda \int_{0}^{1} q(x) d x \leq 0$. Hence the result. 
Lemma 2.11. Let $M$ and $N \in \mathbb{R}^{+}$. If $-\lambda_{0}<\lambda<0$ is such that $-\left(\int_{0}^{1}(1 / p(x)) \int_{0}^{x} q(t) d t d x\right)^{-1}<\lambda \leq$ $-M$ and

$$
(M+\lambda)\left(1+\lambda \int_{0}^{1} \frac{1}{p(x)} \int_{0}^{x} q(t) d t d x\right)-N \lambda \int_{0}^{1} q(x) d x \leq 0,
$$

then for all $x \in[0,1]$,

$$
(M+\lambda) z_{0}(x)-N p(x) z_{0}^{\prime}(x) \leq 0
$$

Proof. Using (2.6) and Remark 2.5, it can be deduced that $z_{0}(x)$ and $p(x) z_{0}^{\prime}(x)$ are decreasing functions of $x$ for $-\lambda_{0}<\lambda<0$, thus

$$
(M+\lambda) z_{0}(x)-N p(x) z_{0}^{\prime}(x) \leq(M+\lambda) z_{0}(1)-N p(1) z_{0}^{\prime}(1) .
$$

Now using (2.6), we get $-p(1) z_{0}^{\prime}(1) \leq(-\lambda) \int_{0}^{1} q(x) d x$ and $z_{0}(1)>1+\lambda \int_{0}^{1}(1 / p(x)) \int_{0}^{x} q(t) d t d x$. This completes the proof.

Note. In Lemma 2.11, we arrive at the integral $\int_{0}^{1}(1 / p(x)) \int_{0}^{x} q(t) d t d x$ which is an improper integral, and it should be convergent. Using the assumption (A2) (iv) and Remark (i) and (ii) at [6, page 434] its convergence can be established.

\section{Well-Ordered Upper and Lower Solutions}

Let us define upper and lower solutions.

Definition 3.1. A function $u_{0} \in C[0,1] \cap C^{2}(0,1]$ is an upper solution of (1.1) if

$$
-\left(p u_{0}^{\prime}\right)^{\prime}+q f\left(x, u_{0}, p u_{0}^{\prime}\right) \geq 0, \quad 0<x<1, u_{0}^{\prime}(0) \leq 0 \leq u_{0}^{\prime}(1)
$$

Definition 3.2. A function $v_{0} \in C[0,1] \cap C^{2}(0,1]$ is a lower solution of (1.1) if

$$
-\left(p v_{0}^{\prime}\right)^{\prime}+q f\left(x, v_{0}, p v_{0}^{\prime}\right) \leq 0, \quad 0<x<1, v_{0}^{\prime}(0) \geq 0 \geq v_{0}^{\prime}(1)
$$

Now, for every $n$, the problem (1.2) has a unique solution $y_{n+1}$ given by (2.5) with $h(x)=$ $-f\left(x, y_{n}, p y_{n}^{\prime}\right)+\lambda y_{n}, A=0$, and $B=0$.

In this section, we show that for the proposed scheme (1.2) a good choice of $\lambda$ is possible so that the solutions generated by the approximation scheme converge monotonically to solutions of (1.1). We require a number of results.

Lemma 3.3. Let $\lambda>0$. If $u_{n}$ is an upper solution of (1.1) and $u_{n+1}$ is defined by (1.2), then $u_{n+1} \leq u_{n}$. 
Proof. Let $w_{n}=u_{n+1}-u_{n}$, then

$$
\begin{aligned}
-\left(p w_{n}^{\prime}\right)^{\prime}+\lambda q w_{n} & =\left(p u_{n}^{\prime}\right)^{\prime}-q f\left(x, u_{n}, p u_{n}^{\prime}\right) \leq 0, \\
w_{n}^{\prime}(0) & \geq 0, \quad w_{n}^{\prime}(1) \leq 0,
\end{aligned}
$$

and using Proposition 2.8, we have $u_{n+1} \leq u_{n}$.

Proposition 3.4. Assume that

(H1) there exist upper solution $\left(u_{0}\right)$ and lower solution $\left(v_{0}\right)$ in $C[0,1] \cap C^{2}(0,1]$ such that $v_{0} \leq u_{0}$ for all $x \in[0,1]$,

(H2) the function $f: D \rightarrow \mathbb{R}$ is continuous on

$$
D:=\left\{\left(x, y, p y^{\prime}\right) \in[0,1] \times R \times R: v_{0} \leq y \leq u_{0}\right\},
$$

(H3) there exists $M \geq 0$ such that for all $\left(x, \tau, p v^{\prime}\right),\left(x, \sigma, p v^{\prime}\right) \in D$,

$$
f\left(x, \tau, p v^{\prime}\right)-f\left(x, \sigma, p v^{\prime}\right) \geq M(\tau-\sigma), \quad(\tau \leq \sigma),
$$

(H4) there exist $N \geq 0$ such that for all $\left(x, u, p v_{1}^{\prime}\right)\left(x, u, p v_{2}^{\prime}\right) \in D$,

$$
\left|f\left(x, u, p v_{1}^{\prime}\right)-f\left(x, u, p v_{2}^{\prime}\right)\right| \leq N\left|p v_{2}^{\prime}-p v_{1}^{\prime}\right| .
$$

Let $\lambda>0$ be such that $\mathcal{\lambda} \geq M\left(1-N \int_{0}^{1} q(x) d x\right)^{-1}$. Then the functions $u_{n+1}$ defined recursively by (1.2) are such that, for all $n \in \mathbb{N}$,

(i) $u_{n}$ is an upper solution of (1.1).

(ii) $u_{n+1} \leq u_{n}$.

Proof. We prove the claims by the principle of mathematical induction. Since $u_{0}$ is an upper solution and by Lemma $3.3 u_{0} \geq u_{1}$; therefore, both the claims are true for $n=0$.

Further, let the claims be true for $n-1$, that is, $u_{n-1}$ is an upper solution and $u_{n-1} \geq u_{n}$. Now, we are required to prove that $u_{n}$ is an upper solution and $u_{n+1} \leq u_{n}$. To prove this, let $w=u_{n}-u_{n-1}$, then we have

$$
-\left(p u_{n}^{\prime}\right)^{\prime}+q f\left(x, u_{n}, p u_{n}^{\prime}\right) \geq p\left[(M-\lambda) w-N\left(\operatorname{sign} w^{\prime}\right) p w^{\prime}\right] .
$$

Thus, to prove that $u_{n}$ is an upper solution, we are required to prove that

$$
(M-\lambda) w-N\left(\operatorname{sign} w^{\prime}\right) p w^{\prime} \geq 0 .
$$

Now, since $w$ satisfies

$$
-\left(p w^{\prime}\right)^{\prime}+\lambda q w=\left(p u_{n-1}^{\prime}\right)^{\prime}-q f\left(x, u_{n-1}, p u_{n-1}^{\prime}\right) \leq 0, \quad w^{\prime(0)} \geq 0, w^{\prime}(1) \leq 0,
$$


from Proposition 2.8, we have $w \leq 0$ for $\lambda>0$. Now, putting the value of $w$ from (2.5) in (3.8), and in view of $h=\left(p u_{n-1}^{\prime}\right)^{\prime}-q f\left(x, u_{n-1}, p u_{n-1}^{\prime}\right) \leq 0$, we deduce that to prove (3.8) it is sufficient to prove that

$$
\begin{aligned}
& (M-\lambda) z_{0}-N\left(\operatorname{sign} w^{\prime}\right) p z_{0}^{\prime} \leq 0 \\
& (M-\lambda) z_{1}-N\left(\operatorname{sign} w^{\prime}\right) p z_{1}^{\prime} \leq 0
\end{aligned}
$$

for all $x \in[0,1]$. Since $z_{1}=z_{0}(1-x)$, using Remark 2.6, the above inequalities will be true if for all $x \in[0,1]$ we have

$$
(M-\lambda) z_{0}(x)+N p(x) z_{0}^{\prime}(x) \leq 0 .
$$

Which is true (Lemma 2.10). Therefore, (3.8) holds, and hence $u_{n}$ is an upper solution.

Now applying Lemma 3.3, we deduce that $u_{n+1} \leq u_{n}$. This completes the proof.

Similarly, we can prove the following two results (Lemma 3.5, Proposition 3.6) for lower solutions.

Lemma 3.5. Let $\lambda>0$. If $v_{n}$ is a lower solution of (1.1) and $v_{n+1}$ is defined by (1.2), then $v_{n} \leq v_{n+1}$.

Proposition 3.6. Assume that (H1), (H2), (H3), and (H4) hold, and let $\lambda>0$ be such that $\lambda \geq$ $M\left(1-N \int_{0}^{1} q(x) d x\right)^{-1}$. Then the functions $v_{n+1}$ defined recursively by (1.2) are such that for all $n \in \mathbb{N}$,

(i) $v_{n}$ is a lower solution of (1.1).

(ii) $v_{n} \leq v_{n+1}$.

In the next result, we prove that upper solution $u_{n}$ is larger than lower solution $v_{n}$ for all $n$.

Proposition 3.7. Assume that (H1), (H2), (H3), and (H4) hold, and let $\lambda>0$ such that $\lambda \geq$ $M\left(1-N \int_{0}^{1} q(x) d x\right)^{-1}$ and for all $x \in[0,1]$

$$
f\left(x, v_{0}, p v_{0}^{\prime}\right)-f\left(x, u_{0}, p u_{0}^{\prime}\right)+\lambda\left(u_{0}-v_{0}\right) \geq 0 .
$$

Then for all $n \in \mathbb{N}$, the functions $u_{n}$ and $v_{n}$ defined recursively by (1.2) satisfy $v_{n} \leq u_{n}$.

Proof. We define a function

$$
h_{i}(x)=f\left(x, v_{i} p v_{i}^{\prime}\right)-f\left(x, u_{i}, p u_{i}^{\prime}\right)+\lambda\left(u_{i}-v_{i}\right), \quad i \in \mathbb{N} .
$$

It is easy to see that for all $i \in \mathbb{N}, w_{i}=u_{i}-v_{i}$ satisfies the following differential equation:

$$
-\left(p w_{i}^{\prime}\right)^{\prime}+\lambda q w_{i}=q\left\{f\left(x, v_{i-1}, p v_{i-1}^{\prime}\right)-f\left(x, u_{i-1}, p u_{i-1}^{\prime}\right)+\lambda\left(u_{i-1}-v_{i-1}\right)\right\}=q h_{i-1} .
$$

Now to prove this proposition again, we use the principle of mathematical induction. For $i=1$, we have $h_{0} \geq 0$, and $w_{1}$ is the solution of (2.1)-(2.2) with $A=0$ and $B=0$. Using Proposition 2.8, we deduce that $w_{1} \geq 0$, that is, $u_{1} \geq v_{1}$. 
Now, let $n \geq 2$, let $h_{n-2} \geq 0$, and let $u_{n-1} \geq v_{n-1}$, then we are required to prove that $h_{n-1} \geq 0$ and $u_{n} \geq v_{n}$. First, we show that for all $x \in[0,1]$ the function $h_{n-1}$ is nonnegative. Indeed, we have

$$
\begin{aligned}
h_{n-1} & =f\left(x, v_{n-1}, p v_{n-1}^{\prime}\right)-f\left(x, u_{n-1}, p u_{n-1}^{\prime}\right)+\lambda\left(u_{n-1}-v_{n-1}\right) \\
& \geq-\left[(M-\lambda) w_{n-1}+N\left(\operatorname{sign} w_{n-1}^{\prime}\right) p w_{n-1}^{\prime}\right] .
\end{aligned}
$$

Here $w_{n-1}$ is a solution of (2.1) with $h(x)=h_{n-2} \geq 0, A=0$, and $B=0$. Arguments similar to Proposition 3.4 can be used to prove that $h_{n-1} \geq 0$. Now, we have $h_{n-1} \geq 0, w_{n}^{\prime}(0)=0$, and $w_{n}^{\prime}(1)=0$, thus from Proposition 2.8, we deduce that $w_{n} \geq 0$, that is, $u_{n} \geq v_{n}$.

Lemma 3.8. If $f\left(x, u, p u^{\prime}\right)$ satisfies (H1), (H2), and

(H5) for all $\left(x, u, p u^{\prime}\right) \in D,\left|f\left(x, u, p u^{\prime}\right)\right| \leq \varphi\left(\left|p u^{\prime}\right|\right)$, where $\varphi:[0, \infty) \rightarrow(0, \infty)$ is continuous and satisfies.

$$
\int_{0}^{\infty} \frac{d s}{\varphi(s)}>\int_{0}^{1} q(x) d x
$$

then there exists $R_{0}>0$ such that any solution of

$$
-\left(p u^{\prime}\right)^{\prime}+q f\left(x, u, p u^{\prime}\right) \geq 0, \quad 0<x<1, u^{\prime}(0)=0=u^{\prime}(1)
$$

with $u \in\left[v_{0}, u_{0}\right]$, for all $x \in[0,1]$, satisfies $\left\|p u^{\prime}\right\|_{\infty}<R_{0}$.

Proof. Consider an interval $\left[x, x_{0}\right] \subset[0,1]$ such that

$$
\forall s \in\left[x, x_{0}\right), \quad u^{\prime}(s)<0, \quad u^{\prime}\left(x_{0}\right)=0 .
$$

Now using (H5), we have

$$
\left(p u^{\prime}\right)^{\prime} \leq q \varphi\left(\left|p u^{\prime}\right|\right)
$$

and after integrating it from $x$ to $x_{0}$ and using (H5), we have

$$
-p u^{\prime} \leq R_{0} .
$$

Similarly for the interval $\left[x_{0}, x\right]$, we have

$$
p u^{\prime} \leq R_{0}
$$

Thus

$$
\left\|p u^{\prime}\right\|_{\infty} \leq R_{0}
$$

In the same way, we can prove the following result for lower solutions. 
Lemma 3.9. If $f\left(x, v, p v^{\prime}\right)$ satisfies (H1), (H2), and (H5), then there exists $R_{0}>0$ such that any solution of

$$
-\left(p v^{\prime}\right)^{\prime}+q f\left(x, v, p v^{\prime}\right) \leq 0, \quad 0<x<1, v^{\prime}(0)=0=v^{\prime}(1)
$$

with $v \in\left[v_{0}, u_{0}\right]$, for all $x \in[0,1]$, satisfies $\left\|p v^{\prime}\right\|_{\infty}<R_{0}$.

Now we are in a situation to prove our final result for the case when upper and lower solutions are well ordered.

Theorem 3.10. Assume (H1), (H2), (H3), (H4), and (H5) are true. Let $\lambda>0$ be such that

$$
\lambda \geq M\left(1-N \int_{0}^{1} q(x) d x\right)^{-1}
$$

and for all $x \in[0,1]$,

$$
f\left(x, v_{0}, p v_{0}^{\prime}\right)-f\left(x, u_{0}, p u_{0}^{\prime}\right)+\lambda\left(u_{0}-v_{0}\right) \geq 0 .
$$

Then the sequences $\left\{u_{n}\right\}$ and $\left\{v_{n}\right\}$ defined by (1.2) converge monotonically to solutions $\tilde{u}(x)$ and $\tilde{v}(x)$ of $(1.1)$. Any solution $z(x)$ of (1.1) in D satisfies

$$
\widetilde{v}(x) \leq z(x) \leq \tilde{u}(x) .
$$

Proof. Using Lemma 3.3 to Lemma 3.9 and Proposition 3.4 to Proposition 3.7, we deduce that the sequences $\left\{u_{n}\right\}$ and $\left\{v_{n}\right\}$ are monotonic $\left(u_{0} \geq u_{1} \geq u_{2} \cdots \geq u_{n} \geq v_{n} \cdots \geq v_{2} \geq v_{1} \geq v_{0}\right)$ and are bounded by $v_{0}$ and $u_{0}$ in $C[0,1]$, and by Dini's theorem, they converge uniformly to $\tilde{u}$ and $\tilde{v}$ (say). We can also deduce that the sequences $\left\{p u_{n}^{\prime}\right\}$ and $\left\{p v_{n}^{\prime}\right\}$ are uniformly bounded and equicontinuous in $C[0,1]$, and by Arzela-Ascoli theorem, there exists uniformly convergent subsequences $\left\{p u_{n_{k}}^{\prime}\right\}$ and $\left\{p v_{n_{k}}^{\prime}\right\}$ in $C[0,1]$. It is easy to observe that $u_{n} \rightarrow \tilde{u}$ and $v_{n} \rightarrow \tilde{v}$ imply $p u_{n}^{\prime} \rightarrow p \tilde{u}^{\prime}$ and $p \widetilde{v}_{n}^{\prime} \rightarrow p \widetilde{v}^{\prime}$.

Solution of (1.2) is given by (2.5) where $h(x)=-f\left(x, y_{n-1}, p y_{n-1}^{\prime}\right)+\lambda y_{n-1}$. Since the sequences are uniformly convergent taking limit as $n \rightarrow \infty$, we get $\tilde{u}$ and $\tilde{v}$ as the solutions of the nonlinear boundary value problem (1.1). Any solution $z(x)$ in $D$ plays the role of $u_{0}$. Hence, $z(x) \geq \widetilde{v}(x)$. Similarly $z(x) \leq \tilde{u}(x)$.

Remark 3.11. When the source function is derivative independent, that is, $N=0$, in this case we can choose $\lambda=M$. 


\section{Upper and Lower Solutions in Reverse Order}

In this section, we consider the case when the upper and lower solutions are in reverse order, that is,

$$
u_{0}(x) \leq v_{0}(x)
$$

For this, we require opposite one-sided Lipschitz condition, and we assume that

$(F 1)$ there exists upper solution $\left(u_{0}\right)$ and lower solution $\left(v_{0}\right)$ in $C[0,1] \cap C^{2}(0,1]$ such that $u_{0} \leq v_{0}$ for all $x \in[0,1]$,

$(F 2)$ the function $f: D_{0} \rightarrow \mathbb{R}$ is continuous on

$$
D_{0}:=\left\{\left(x, y, p y^{\prime}\right) \in[0,1] \times R \times R: u_{0} \leq y \leq v_{0}\right\},
$$

(F3) there exists $M \geq 0$ such that for all $\left(x, \tilde{\tau}, p v^{\prime}\right),\left(x, \widetilde{\sigma}, p v^{\prime}\right) \in D_{0}$,

$$
f\left(x, \tilde{\sigma}, p v^{\prime}\right)-f\left(x, \tilde{\tau}, p v^{\prime}\right) \geq-M(\tilde{\sigma}-\tilde{\tau}), \quad(\tilde{\tau} \leq \tilde{\sigma}),
$$

(F4) there exist $N \geq 0$ such that for all $\left(x, u, p v_{1}^{\prime}\right)\left(x, u, p v_{2}^{\prime}\right) \in D_{0}$,

$$
\left|f\left(x, u, p v_{1}^{\prime}\right)-f\left(x, u, p v_{2}^{\prime}\right)\right| \leq N\left|p v_{2}^{\prime}-p v_{1}^{\prime}\right| .
$$

Here again we define the approximation scheme by (1.2) and use the Antimaximum principle. We make a good choice of $\lambda$ so that the sequences thus generated converge to the solution of the nonlinear problem. Similar to Section 3, we require the following lemmas and propositions.

Lemma 4.1. Let $-\lambda_{0}<\lambda<0$. If $u_{n}$ is an upper solution of (1.1) and $u_{n+1}$ is defined by (1.2), then $u_{n+1} \geq u_{n}$.

Proof. Let $w_{n}=u_{n+1}-u_{n}$, then

$$
\begin{aligned}
-\left(p w_{n}^{\prime}\right)^{\prime}+\lambda q w_{n} & =\left(p u_{n}^{\prime}\right)^{\prime}-q f\left(x, u_{n}, p u_{n}^{\prime}\right) \leq 0, \\
w_{n}^{\prime}(0) & \geq 0, \quad w_{n}^{\prime}(1) \leq 0,
\end{aligned}
$$

and using Proposition 2.8, we have $u_{n+1} \geq u_{n}$.

Proposition 4.2. Assume that (F1), (F2), (F3), and (F4) hold. Let $-\lambda_{0}<\lambda<0$ be such that $-\left(\int_{0}^{1}(1 / p(x)) \int_{0}^{x} q(t) d t d x\right)^{-1}<\lambda \leq-M$ and $(M+\lambda)\left(1+\lambda \int_{0}^{1}(1 / p(x)) \int_{0}^{x} q(t) d t d x\right)-$ $N \lambda \int_{0}^{1} q(x) d x \leq 0$. Then the functions $u_{n+1}$ defined recursively by (1.2) are such that, for all $n \in \mathbb{N}$,

(i) $u_{n}$ is an upper solution of (1.1);

(ii) $u_{n+1} \geq u_{n}$. 
Proof. Using Remarks 2.5 and 2.7, Lemmas 2.11 and 4.1, and on the lines of the proof of Proposition 3.4, this proposition can be deduced easily.

In the same way, we can prove the following results for the lower solutions.

Lemma 4.3. Let $-\lambda_{0}<\lambda<0$. If $v_{n}$ is a lower solution of (1.1) and $v_{n+1}$ is defined by (1.2), then $v_{n} \geq v_{n+1}$.

Proposition 4.4. Assume that (F1), (F2), (F3), and (F4) hold. Let $-\lambda_{0}<\lambda<0$ be such that $-\left(\int_{0}^{1}(1 / p(x)) \int_{0}^{x} q(t) d t d x\right)^{-1}<\lambda \leq-M$ and $(M+\lambda)\left(1+\lambda \int_{0}^{1}(1 / p(x)) \int_{0}^{x} q(t) d t d x\right)-$ $N \lambda \int_{0}^{1} q(x) d x \leq 0$. Then the functions $v_{n+1}$ defined recursively by (1.2) are such that, for all $n \in \mathbb{N}$,

(i) $v_{n}$ is a lower solution of (1.1);

(ii) $v_{n} \geq v_{n+1}$.

In the next result, we prove that lower solution $v_{n}$ is larger than upper solution $u_{n}$ for all $n$.

Proposition 4.5. Assume that (F1), (F2), (F3), and (F4) hold. Let $-\lambda_{0}<\ell<0$ be such that $-\left(\int_{0}^{1}(1 / p(x)) \int_{0}^{x} q(t) d t d x\right)^{-1}<\lambda \leq-M$ and

$$
(M+\lambda)\left(1+\lambda \int_{0}^{1} \frac{1}{p(x)} \int_{0}^{x} q(t) d t d x\right)-N \lambda \int_{0}^{1} q(x) d x \leq 0,
$$

and for all $x \in[0,1]$,

$$
f\left(x, v_{0}, p v_{0}^{\prime}\right)-f\left(x, u_{0}, p u_{0}^{\prime}\right)+\lambda\left(u_{0}-v_{0}\right) \geq 0 .
$$

Then, for all $n \in \mathbb{N}$, the functions $u_{n}$ and $v_{n}$ defined recursively by (1.2) satisfy $v_{n} \geq u_{n}$.

Now similar to Lemmas 3.8 and 3.9, we state the following two results. These results establish a bound on $p(x) u^{\prime}(x)$ and $p(x) v^{\prime}(x)$.

Lemma 4.6. If $f\left(x, u, p u^{\prime}\right)$ satisfies $(F 1),(F 2)$, and

(F5) for all $\left(x, u, p u^{\prime}\right) \in D_{0},\left|f\left(x, u, p u^{\prime}\right)\right| \leq \varphi\left(\left|p u^{\prime}\right|\right)$, where $\varphi:[0, \infty) \rightarrow(0, \infty)$ is continuous and satisfies

$$
\int_{0}^{\infty} \frac{d s}{\varphi(s)}>\int_{0}^{1} q(x) d x
$$

then there exists $R_{0}>0$ such that any solution of

$$
-\left(p u^{\prime}\right)^{\prime}+q f\left(x, u, p u^{\prime}\right) \geq 0, \quad 0<x<1, u^{\prime}(0)=0=u^{\prime}(1)
$$

with $u \in\left[u_{0}, v_{0}\right]$, for all $x \in[0,1]$, satisfies $\left\|p u^{\prime}\right\|_{\infty}<R_{0}$. 
Lemma 4.7. If $f\left(x, v, p v^{\prime}\right)$ satisfies $(F 1),(F 2)$, and (F5), then there exists $R_{0}>0$ such that any solution of

$$
-\left(p v^{\prime}\right)^{\prime}+q f\left(x, v, p v^{\prime}\right) \leq 0, \quad 0<x<1, v^{\prime}(0)=0=v^{\prime}(1)
$$

with $v \in\left[u_{0}, v_{0}\right]$, for all $x \in[0,1]$, satisfies $\left\|p v^{\prime}\right\|_{\infty}<R_{0}$.

Finally we arrive at the theorem similar to Theorem 3.10.

Theorem 4.8. Assume (F1), (F2), (F3), (F4), and (F5) are true. Let $-\lambda_{0}<\lambda<0$ be such that $-\left(\int_{0}^{1}(1 / p(x)) \int_{0}^{x} q(t) d t d x\right)^{-1}<\lambda \leq-M$ and $(M+\lambda)\left(1+\lambda \int_{0}^{1}(1 / p(x)) \int_{0}^{x} q(t) d t d x\right)-$ $N \lambda \int_{0}^{1} q(x) d x \leq 0$, and for all $x \in[0,1]$,

$$
f\left(x, v_{0}, p v_{0}^{\prime}\right)-f\left(x, u_{0}, p u_{0}^{\prime}\right)+\lambda\left(u_{0}-v_{0}\right) \geq 0 .
$$

Then the sequences $\left\{u_{n}\right\}$ and $\left\{v_{n}\right\}$ defined by (1.2) converge monotonically to solutions $\tilde{u}(x)$ and $\tilde{v}(x)$ of (1.1). Any solution $z(x)$ of (1.1) in $D_{0}$ satisfies

$$
\widetilde{u}(x) \leq z(x) \leq \widetilde{v}(x)
$$

Proof. Using Lemma 4.1 to Lemma 4.7 and Proposition 4.2 to Proposition 4.5, we deduce that

$$
u_{0} \leq u_{1} \leq u_{2} \leq \cdots \leq u_{n} \leq v_{n} \cdots \leq v_{1} \leq v_{0}
$$

Now similar to the proof of Theorem 3.10, the result of this theorem can be deduced.

Remark 4.9. When the source function is derivative independent, that is, $N=0$, in this case we can choose $\lambda=-M$.

\section{Conclusion}

We establish some existence results under quite general conditions on $p(x), q(x)$, and $f\left(x, y, p y^{\prime}\right)$. We prove some fundamental differential inequalities which enables us to prove the monotonicity of the sequences $\left\{u_{n}\right\}$ and $\left\{v_{n}\right\}$. For this we have analyzed the singular differential equation $-\left(p y^{\prime}\right)^{\prime}+\lambda q y=0$ and derived properties of the solutions and their derivatives. This work generalizes our previous work [3]. Lot of exploration is still left. For example, one can consider different type of boundary conditions, and one can also try to remove the Lipschitz condition.

\section{References}

[1] Y. Zhang, "Positive solutions of singular sublinear Dirichlet boundary value problems," SIAM Journal on Mathematical Analysis, vol. 26, no. 2, pp. 329-339, 1995.

[2] D. O'Regan and M. A. El-Gebeily, "Existence, upper and lower solutions and quasilinearization for singular differential equations," IMA Journal of Applied Mathematics, vol. 73, no. 2, pp. 323-344, 2008. 
[3] A. K. Verma, "The monotone iterative method and zeros of Bessel functions for nonlinear singular derivative dependent BVP in the presence of upper and lower solutions," Nonlinear Analysis, Theory, Methods and Applications, vol. 74, no. 14, pp. 4709-4717, 2011.

[4] M. Cherpion, C. de Coster, and P. Habets, "A constructive monotone iterative method for second-order BVP in the presence of lower and upper solutions," Applied Mathematics and Computation, vol. 123, no. 1, pp. 75-91, 2001.

[5] C. de Coster and P. Habets, Two-Point Boundary Value Problems: Lower and Upper Solutions, vol. 205 of Mathematics in Science and Engineering, Elsevier, Amsterdam, The Netherlands, 2006.

[6] D. O'Regan, "Existence theory for nonresonant singular boundary value problems," Proceedings of the Edinburgh Mathematical Society, vol. 38, no. 3, pp. 431-447, 1995.

[7] E. C. Titchmarsh, Eigen Function Expansion. Part I, Oxford University Press, Oxford, UK, 1962. 


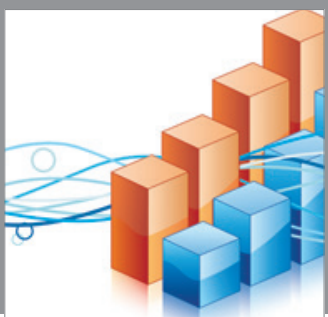

Advances in

Operations Research

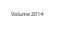

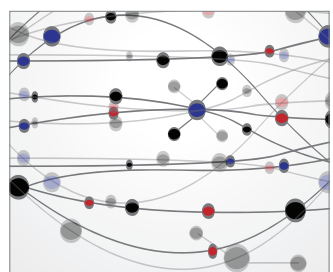

\section{The Scientific} World Journal
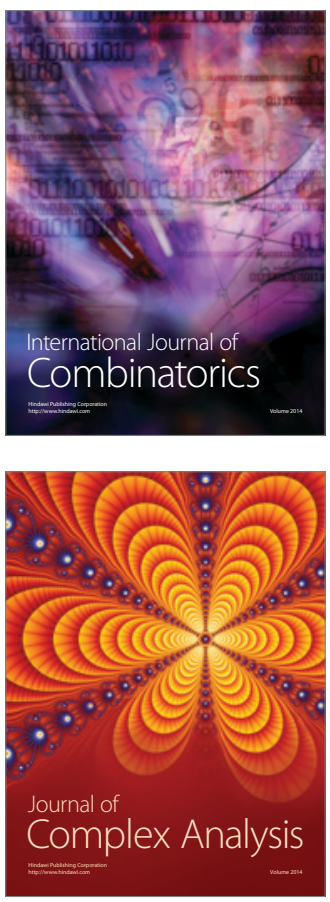

International Journal of

Mathematics and

Mathematical

Sciences
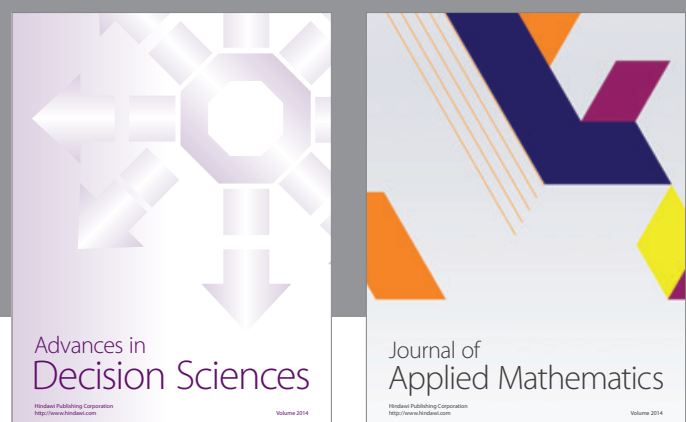

Journal of

Applied Mathematics
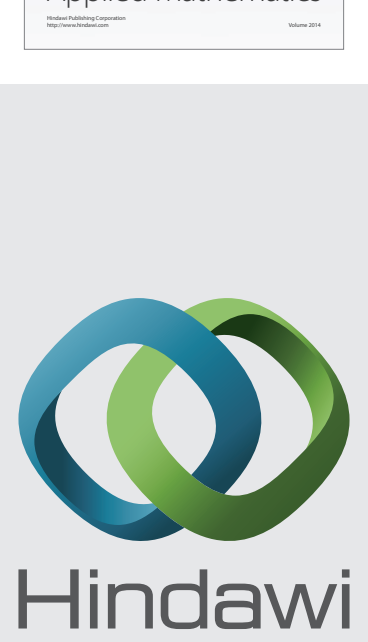

Submit your manuscripts at http://www.hindawi.com
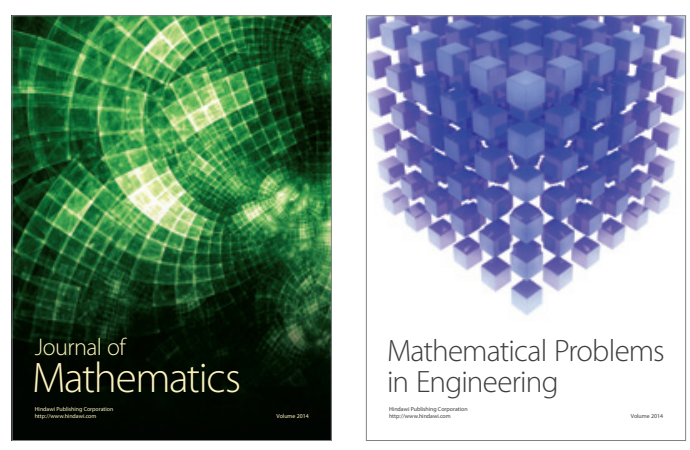

Mathematical Problems in Engineering
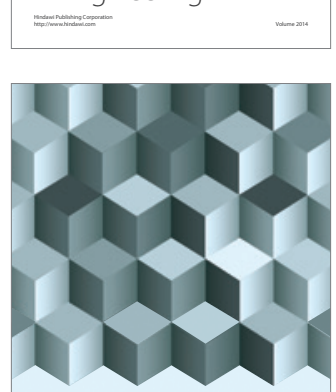

Journal of

Function Spaces
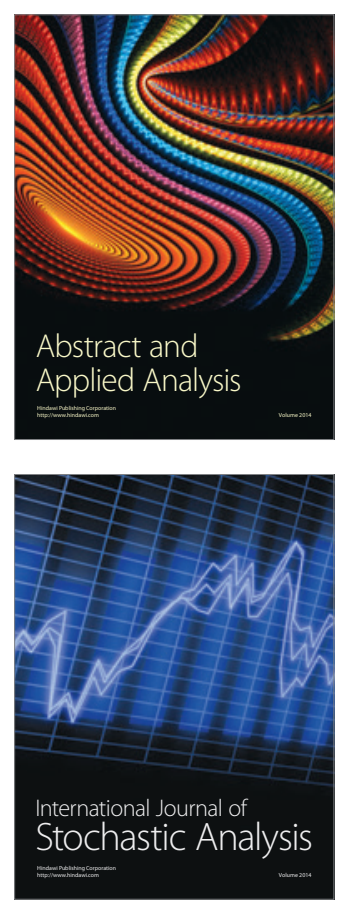

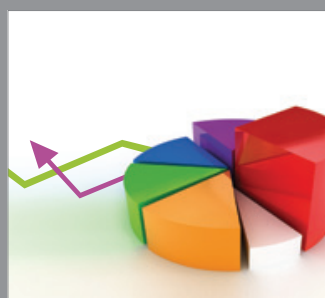

ournal of

Probability and Statistics

Promensencen
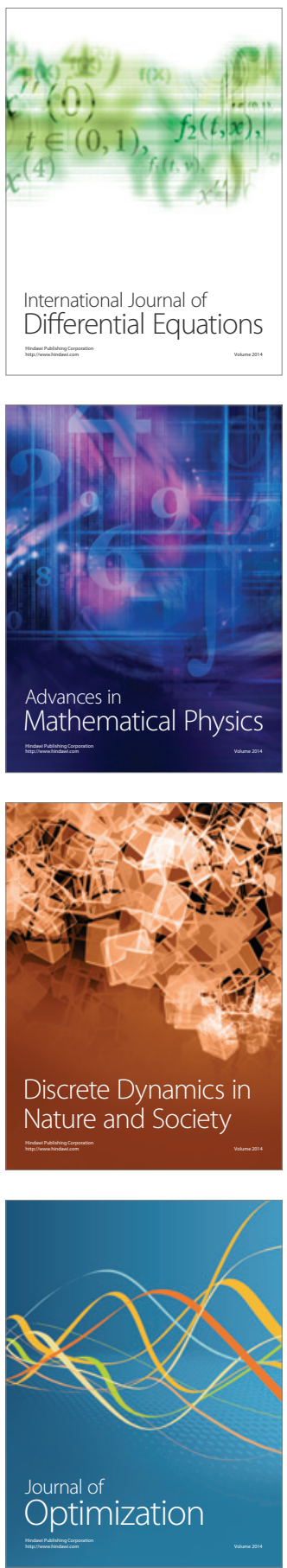